\title{
Imaginarios sociales y las 'nubes de palabras'. Elementos clave en la construcción de redes grupales de aprendizaje
}

Social imaginaries and 'word clouds'. Key elements in the construction of group learning networks

Citar como: Cano M., J. J. (2020). Imaginarios sociales y las 'nubes de palabras'. Elementos clave en la construcción de redes grupales de aprendizaje. CITAS, 6(1). https://doi.org/10.15332/24224529.6355

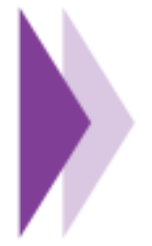

Jeimy J. Cano M. ${ }^{1}$

1 Universidad de los Andes

Correo electrónico: jcano@uniandes.edu.co 


\section{Resumen}

La acelerada convergencia tecnológica, la necesidad de generar nuevos conocimientos y anticiparse a un contexto volátil, incierto, complejo y ambiguo, establece retos clave tanto para las instituciones de educación superior, como para los escenarios empresariales. En este sentido, se hace necesario habilitar nuevos modos de conexión y relaciones para desarrollar y consolidar redes grupales de aprendizaje (en extensión a las redes personales de aprendizaje), con el fin de crear territorios propicios para aprender. Lo anterior tendrá mayor alcance si se apoya en mediaciones tecnológicas, como las nubes de palabras, para revelar los imaginarios sociales propios de una comunidad alrededor de una temática específica. En consecuencia, este artículo hace evidente la emergencia de propiedades sistémicas propias de la representación de la información para identificar nuevas correlaciones significativas de aprendizaje, fruto de una lectura colectiva que sintoniza personas, imaginarios y medios tecnológicos. Para ello, se presenta el resultado de ejercicios de elaboración de nubes de palabras realizados con diferentes comunidades de personas sobre distintos temas, en las que se hacen evidentes sus posturas y donde se funda así un escenario de convergencia de visiones personales y patrones emergentes.

Palabras clave: imaginarios sociales, nubes de palabras, redes personales de aprendizaje, pensamiento sistémico, redes grupales de aprendizaje.

En un contexto de transformaciones aceleradas, de revoluciones personales e industriales, que manifiestan el espíritu de cambio en la humanidad, las contradicciones, inestabilidades y, algunas veces, sin sentidos alertan sobre las nuevas lecturas que se hacen y se pueden hacer sobre la interacción social y humana. De este modo, las dinámicas sociales habilitan escenarios de aprendizaje, de cambios de comportamientos que se experimentan cada vez que se "suspende el entendimiento conocido de la realidad" (Reyes y Zarama, 1998).

Si "el aprendizaje no se puede diseñar" (Gros y Mas, 2016, p. 66) —dado que pertenece al ámbito de la experiencia y de la práctica一, la sociedad, consciente de esta misión y reto de supervivencia, debe anticipar el proceso que deberá seguir cada uno de sus participantes para seleccionar aquellos contenidos que son de interés y relevancia, con el fin de organizarlos y así motivar el aprendizaje de sus miembros como una capacidad para cuestionar su propia realidad y construir distinciones que no existían.

$\mathrm{Si}$ "solo se aprende cuando aparece un comportamiento nuevo" (Barreda, 1995, p. 1), la pregunta es: ¿cuáles son los comportamientos que requerimos para mantener el desarrollo asimétrico y armónico al mismo tiempo en el contexto social? En otras palabras, ¿qué aprendizajes debemos alcanzar para desconectar las preconcepciones existentes y así incorporar nuevas formas de ver el mundo, nuevos patrones de comportamientos, que eleven el nivel de entendimiento de lo social y lo humano como prerrequisito de las sinergias sociales requeridas para transformar la realidad?

En ese sentido, las redes personales de aprendizaje (Castañeda y Adell, 2013) que sitúan su foco en la manera como las personas aprenden y crean su propio entorno personalizado para explorar y retar sus saberes previos, se configuran como una respuesta a las nuevas exigencias del entorno social, con el fin de encontrar formas alternas de conectar y construir saberes flexibles para

\section{Abstract}

The accelerated technological convergence, the need to generate new knowledge and to anticipate a volatile, uncertain, complex and ambiguous context, establishes key challenges for both higher education institutions and business scenarios. In this sense, it is necessary to enable new modes of connection and relationships to develop and consolidate group learning networks (as an extension of personal learning networks), in order to create territories conducive to learning. The foregoing will have greater scope if technological mediations, such as word clouds, are used to reveal the social imaginaries of a community around a specific topic. Consequently, this article makes evident the emergence of systemic properties of the representation of information, to identify new significant learning correlations, the result of a collective reading that tunes people, imaginations and technological media. To this end, the results of word cloud elaboration exercises carried out with different communities of people on different topics is presented, in which their positions become evident and where a scenario of convergence of personal visions and emerging pattern is thus founded.

Keywords: social imaginaries, word clouds, personal learning networks, systems theory, group learning networks.

crear sinergias mediante el uso eficiente de las tecnologías de la información.

Así las cosas, las nubes de palabras, como mediación tecnológica ${ }^{1}$, facilitan el aprendizaje a través de la colaboración, es decir, construcción colectiva de los diferentes participantes que incluye diversos puntos de vista y posturas sobre un tema en particular (Barkley, Cross y Major, 2012), y habilita el surgimiento de una red grupal de aprendizaje (RGA) como un marco base para revelar los imaginarios sociales propios de comunidades específicas, los cuales permiten una puesta en común de saberes previos y una vista holística ${ }^{2}$ sobre la comprensión de una temática de interés.

En consecuencia, para dar cuenta de los retos que implican las redes grupales de aprendizaje, este documento desarrolla en primer lugar una aproximación epistemológica basada en la sociocibernética, como fundamento del entendimiento de la complejidad de los fenómenos sociales, para luego comprender los imaginarios sociales que se articulan desde una realidad específica. Luego, se introducen las nubes de palabras como herramienta tecnológica para motivar el aprendizaje, reflexiones que serán articuladas con el concepto de redes grupales de aprendizaje, como fenómeno emergente que sintoniza personas, imaginarios y medios tecnológicos.

\section{Fundamento epistemológico}

Los sistemas sociales emergen "cuando miembros de un colectivo producen una red cerrada de interacciones o relaciones recurrentes" (Espejo, 2012, p. 329), donde comparten significados y experiencias gracias a sus acciones y decisiones, que los hacen distintos de otros colectivos, dándoles identidad propia.

Lo anterior representa que los significados creados y producidos por los actores de la comunidad son determinados por 
sus estructuras y no necesariamente por la información que se encuentra en el medio donde esta opera (Espejo, 2012, p. 331). Esto es que existe una red de significados compartidos que reconoce la información del medio donde se encuentra el sistema social, para incorporarla en la medida en que sea pertinente y así mantener la identidad de dicha comunidad. Esta dinámica, reconocida desde la sociocibernética como "la ciencia de los sistemas en sociología y otras ciencias sociales", la cual se "centra en la resolución de problemas" (Maas et ál., 2012, p. 26) propios de la dinámica social, establece que "todo conocimiento es inevitablemente una construcción interna de un sistema, que se basa en operaciones que captan la realidad bajo la forma de distinciones" (Corsi et ál., 1996, p. 51).

La sociocibernética pone el énfasis en el observador, en cada uno de los participantes del tejido social analizado, quien es el que construye y reconstruye la realidad a partir de la diferenciación y de la distinción de elementos y relaciones, y que, consciente de su participación en las interacciones sociales, desarrolla su identidad que lo distingue e integra al mismo tiempo para transformarse a sí mismo y al sistema en el cual participa (Maas et ál., 2012).

La adaptación de los sistemas sociales transita un proceso de aprendizaje y desaprendizaje (Pourdehnad et ál., 2006) que requiere una alta inversión de energía de sus participantes y relaciones. Sin embargo, para que este proceso ocurra, nuevas distinciones se deben identificar y traducirse en un nuevo repertorio de posibles acciones. Cuando esto acontece, se dice que el sistema se ha movido a un nivel superior, dado que ha desarrollado nuevas prácticas en un dominio particular, recuperando su seguridad para actuar en ese dominio (Reyes y Zarama, 1998).

La sociocibernética entiende los conglomerados sociales como sistemas autoorganizados y autorreferenciados, de tal forma que generan la variedad requerida para descubrir y reconocer el mundo, y así dar la respuesta más adecuada a los retos del entorno. De esta manera, el sistema se acopla con el ambiente para evolucionar y adaptarse, con el fin de sobrevivir y mantenerse vigente a pesar de las inestabilidades e inciertos que se presenten en la construcción de sus propias respuestas.

En consecuencia, esta dinámica social, que leída desde la sociocibernética implica el reconocimiento de cada uno de los actores y sus diferentes redes personales de aprendizaje como fundamento de la construcción de nuevos conocimientos, permite comprender los retos del entorno para descubrir nuevas distinciones y actualizar los saberes disponibles como una propiedad emergente que repiensa y reinventa las relaciones de los participantes.

\section{Los imaginarios sociales}

En línea con lo anterior, los imaginarios sociales, entendidos como aquellas construcciones sociales que establecen una mediación entre lo que se asume como realidad y lo que se percibe, hacen visible lo invisible (Cegarra, 2012), crean comportamientos regulados que son reconocidos e integrados a la dinámica social para motivar una experiencia colectiva, y habilitan la generación de conocimiento que permite resignificar sus relaciones para mantenerse vigente en un entorno cambiante e inestable.

Los imaginarios sociales, como categoría envolvente de la realidad social, crean una vista convergente de posibles conversaciones antagónicas que permiten elaborar distinciones a partir del reconocimiento de la diferencia. De esta forma, se construye sentido alrededor de una temática de interés no con el fin de crear posiciones opuestas, sino para visualizar puntos de vista diferentes que articulan y se aglomeran alrededor de un propósito común, que es fuente de sentido y significado para cada uno de los participantes.

De acuerdo con Pintos (1995), los imaginarios sociales se entienden desde el constructivismo sistémico como mecanismos de comprensión de la realidad y del orden social. Por lo tanto, este investigador define los imaginarios sociales como "aquellos esquemas, construidos socialmente, que nos permiten percibir algo como real, explicarlo e intervenir operativamente en lo que en cada sistema social se considere como realidad", definición que supone:

- "La realidad social es el resultado de las distinciones efectuadas por observadores que operan con unas particulares (y siempre parciales) observaciones" (Carretero, 2010, p. 100).

- "Un determinado imaginario social, al operar mediante una distinción, haría relevante una concreta manera de ser de la realidad, haciendo opacas otras posibles maneras de ser \#también plausibles\# a través de las cuales se nos podría hacer presente esa misma realidad" (p. 100).

- "Las sociedades actuales son sociedades policontextuales, donde existe una prevalencia de la competencia entre distintas instituciones, medios de comunicación, entre otros, por adueñarse de una definición particular de la realidad" (p. 101).

De esta forma, la convergencia de las redes personales de aprendizaje, como fenómenos sociales y educativos, generan espacios conjuntos donde nuevos saberes se revelan, alrededor de una construcción social con intereses compartidos, de tal forma que puedan integrar tanto aprendizajes formales como informales, alrededor de constructos conceptuales que no son estáticos, los cuales se modifican conforme se nutran las interacciones entre las redes personales, para dar paso a la emergencia de las RGA.

\section{Las 'nubes de palabras' como mediación tecnológica para el aprendizaje}

De acuerdo con Gómez, García y Therón (2014), una nube de palabras o nubes semánticas de etiquetas es una herramienta de analítica de datos que revela un conjunto ordenado de etiquetas, ponderadas visualmente dependiendo de su popularidad y cuyo tamaño en la representación corresponde con su frecuencia de uso.

El uso de la nube de palabras en un conjunto de personas con intereses semejantes pone en evidencia sus reflexiones y conceptos más significativos. Esta interacción vincula cada uno de los intereses particulares y sus diversas posturas, bien convergentes o divergentes, para desarrollar una comprensión compartida que revela no solo los saberes previos de dicho conjunto, sino sus imaginarios sociales vigentes sobre problemáticas y retos comunes.

Las nubes de palabras, si bien no fueron pensadas para favorecer procesos de construcción de conocimiento, ni elaboradas con fundamentos formativos (Gros, 2008), sí son habilitadores educativos que además de motivar una construcción colaborativa de saberes, reconocen las propuestas particulares de cada participante y sus interacciones para canalizar un nuevo flujo de aprendizaje en red que desarrolla escenarios impregnados de "transversalidad y transmedialidad"3 (Llorens, 2016, p. 149).

En ese sentido, las nubes de palabras fundan un nuevo territorio de aprendizaje mediado por tecnología de analítica de datos, que genera una "representación que utiliza mecanismos expresivos para obtener un conocimiento holístico e interconectado de la 
realidad representada, implicando emocionalmente al espectador en su comprensión" (Llorens, 2016, p. 152).

Esta visualización gráfica de la dinámica de conocimiento personal, ahora en una lectura colectiva, determina nuevos contextos de aprendizaje en grupos y equipos donde se advierten correlaciones significativas entre las diferentes etiquetas utilizadas, haciendo evidente la emergencia de propiedades sistémicas de este ejercicio, donde se descubren distinciones inéditas o enriquecidas y "el aprendizaje se traduce en esa evaluación hecha por los observadores basada en un cambio en las capacidades comprobadas para tomar acción" (Ison, 2017, p. 104).

De esta manera, las nubes de palabras, como herramienta tecnológica, faculta a grupos sociales específicos para favorecer procesos de interacción y solución conjunta de inquietudes, creando un escenario tecnológicamente modificado de aprendizaje colaborativo, donde ninguno de los participantes se considera aislado, y los objetivos y responsabilidades de estos se condensan y realizan en una interacción entre iguales que comparten experiencia y conocimiento (Gros, 2008).

\section{Las redes grupales de aprendizaje y las nubes de palabras}

Si se entiende como una red personal de aprendizaje "al conjunto de herramientas virtuales, personas, contextos y plataformas, que permiten a un individuo construir su propio proceso de aprendizaje, en incluso representarlo gráficamente" (Llorens, 2016, p. 147), una Red Grupal de Aprendizaje (RGA) será el resultado de la interacción de las diferentes redes personales de aprendizaje, como una propiedad emergente de un sistema de interacción de los participantes de una comunidad alrededor de una temática específica.

Esta RGA, como sistema autoorganizado y autorreferenciado, se configura y se renueva basado en una perspectiva constructivista, donde la diferencia es lo que permite dar forma a una visión emergente de los nuevos saberes que son requeridos por la comunidad. La producción de conocimiento, fruto de las relaciones entre pares, configura una inteligencia colectiva que define un entramado de acciones cognitivas que revelan una red invisible y emergente de aprendizajes para dar cuenta del reto conceptual que supone un tema de interés particular.

Luego, una red grupal de aprendizaje, como dinámica emergente que sintoniza personas, imaginarios y medios tecnológicos, deviene en una perspectiva sistémica (sociocibernética) que profundiza en las estructuras de las relaciones creadas entre las redes personales de aprendizaje para comprender el funcionamiento y la funcionalidad del sistema, a partir del entendimiento de su complejidad inherente para alcanzar la variedad requerida que armonice la estabilidad e inestabilidad, con organización y el caos.

Así las cosas, el uso de las nubes de palabras generadas por la participación de grupos específicos de personas crean entornos de aprendizaje, revelan imaginarios sociales y procesos cognitivos elaborados por sus propios participantes, que hacen evidente una red grupal de aprendizaje, donde se enriquecen las relaciones entre pares, se renuevan estructuras de conocimiento y se actualizan los saberes previos de sus actores en función de un contexto que da sentido a sus acciones.

A manera de ejemplo se presentan los resultados del uso de las nubes de palabras al utilizar los servicios de una plataforma en línea denominada Mentimeter ${ }^{4}$, desde donde se lanzaron diversos ejercicios con comunidades distintas, a través de los cuales se hizo evidente un imaginario social particular para un tema, y se configuró una visión y comprensión base de una temática de interés que terminó por configurar un compromiso de aprendizaje colectivo, representado en los resultados y reflexiones posteriores, donde se interrogan los saberes previos y se amplían las opciones de experiencia práctica para aprender de manera colaborativa. 
Figura 1. Nube de palabras 113 participantes (padres de familia)

\section{Frente al reto de la crianza en un contexto digital ¿qué palabras vienen a su mente?}

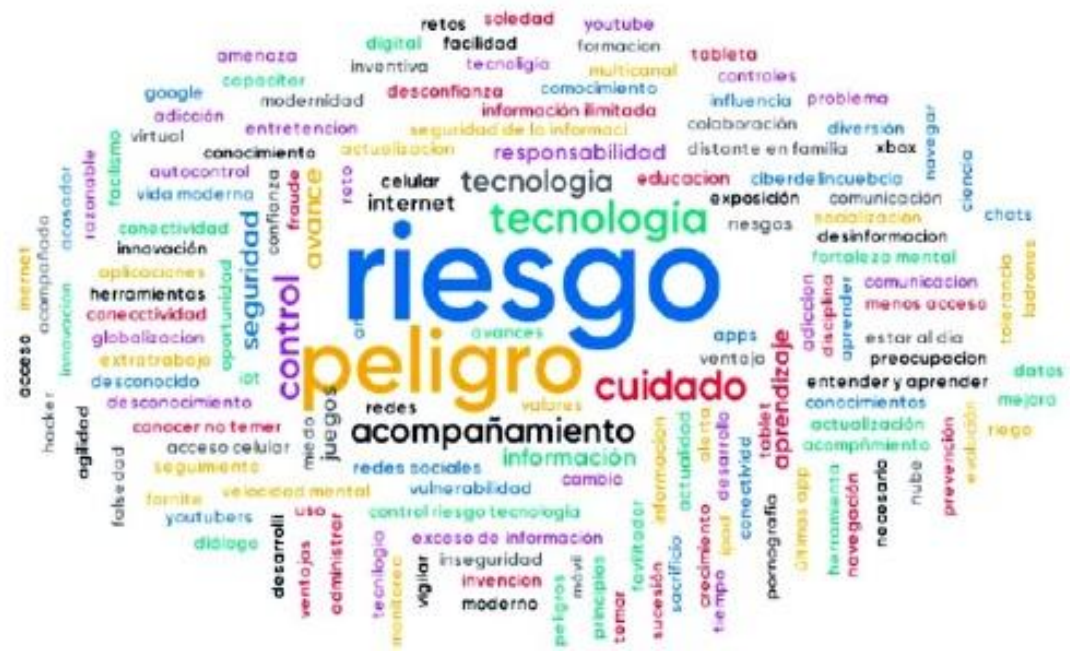

Fuente: elaboración propia.

Figura 2. Nube de palabras de 64 participantes (profesionales sector financiero en Latinoamérica)

\section{¿Para usted qué es transformación digital?}

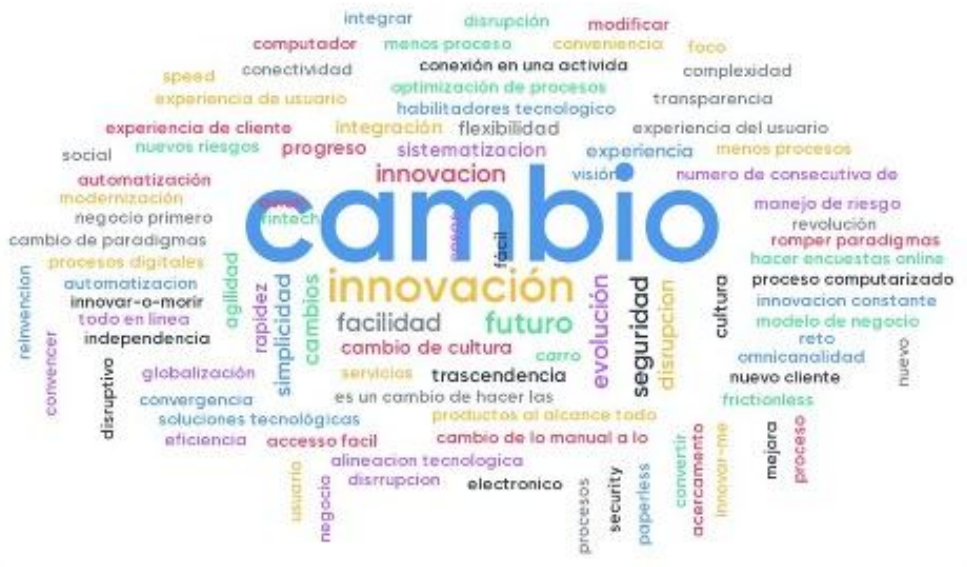

Fuente: elaboración propia.

El ejercicio realizado con padres de familia de un colegio de clase media\#alta en la ciudad de Bogotá, como se observa en la figura 1, buscaba establecer el referente propio de esa comunidad respecto a la crianza en el contexto digital. Si bien 
existen distintas vistas de este reto en las familias, motivar una vista convergente y común permitió construir una visión compartida desde las diferencias. Esto se tradujo en la conformación de una red grupal de aprendizaje, como consecuencia de la interacción de todos los participantes y sus redes personales de aprendizaje. Esto le permitió al colegio establecer el imaginario base de los padres de familia, con el cual se articularon las acciones requeridas para apoyarlos y acompañarlos en este reto de la crianza en el contexto digital.

De otra parte, en la figura 2 se advierte el resultado de la aplicación de un ejercicio semejante al anterior, focalizado en comprender qué es una transformación digital en el sector financiero. El reto de la pregunta en un escenario de participación latinoamericana y del Caribe implica concretar, desde diferentes culturas y prácticas empresariales, cómo estas entidades deben reinventarse y poner atención a las condiciones requeridas para que lo digital tome forma y no se traduzca en un discurso estéril, que no moviliza las fuerzas culturales para concretar ese momentum que las organizaciones requieren para entrar en la ola digital.

Los dos resultados revelan los imaginarios de los participantes en las temáticas específicas, quienes, habilitados desde sus redes personales de aprendizaje, establecen un marco común de comunicación que le permite a la red grupal de aprendizaje emergente concretar nuevos elementos de aprendizaje que se construyen desde la diferencia y las reflexiones propias de cada uno de los individuos involucrados en el ejercicio.

\section{Conclusiones}

Si aprender consiste, de acuerdo con García (2018), en descubrir el acontecimiento que se nos presenta y con frecuencia se esconde envuelto en los hechos, las redes personales de aprendizaje fundan un medio concreto y práctico para explorar y navegar en un mar de información, con el fin de concretar nuevas fuentes de conocimiento y cuestionar los saberes previos.

De esta forma, si entendemos, como afirma Varela, Thompson y Rosch (1997), que el "conocimiento es el resultado de una interpretación que emerge de nuestra capacidad de comprensión" (p. 177), se está reconociendo la relación inherente que cada persona y organización tiene con su entorno y, por la tanto, la riqueza conceptual y analítica que se tiene para repensar todo aquello que se hace. En ese sentido, como afirma Gros (2008), "el conocimiento no solo se construye de forma individual en la mente del sujeto, sino que hay una construcción dinámica y cambiante de origen social y cultural" (p. 54), que configura una red grupal de aprendizaje que emerge como respuesta a un interés particular de una comunidad.

En este contexto, las nubes de palabras, como habilitador tecnológico del aprendizaje, integran los imaginarios sociales de los participantes, habilitan un nuevo escenario de comprensión de la realidad y sintonizan los intereses individuales, para desarrollar una dinámica de grupos. Esta se representa en una red grupal de aprendizaje que reconoce a los otros en la diferencia, para integrarlo en una lectura de inteligencia colectiva que responde a los intereses mutuos alrededor de temáticas específicas.

En consecuencia, las redes grupales de aprendizaje, habilitadas a través de las nubes de palabras, evidencian la presencia de un imaginario social y establecen un recurso educativo emergente. Este permite un aprendizaje basado en red, donde se crean distinciones que "suspenden la realidad" (Reyes y Zarama, 1998) para recorrer una espiral ascendente de conocimiento, que se inicia en una red personal de aprendizaje, y continúa y evoluciona en cada interacción de sus participantes al retar y renovar el conjunto de saberes previos y así procurar el acoplamiento estructural (Maturana y Varela, 1999) con el entorno.

\section{Referencias}

Barkley, E., Cross, K. P. y Major, C. (2012). Técnicas de aprendizaje colaborativo. Manual para el profesorado universitario (2. ${ }^{a}$ ed.). Ministerio de Educación, Cultura y Deporte. Ediciones Morata.

Barreda, R. (1995). Aprendizaje. La función de educación en la empresa moderna. Conorg.

Carretero, A. (2010). Para una tipología de las representaciones sociales. Una lectura de sus implicaciones epistemológicas. EMPIRIA. Revista de Metodología de Ciencias Sociales, (20), 88\#108. http://www.redalyc.org/articulo.oa?id=297125195004

Castañeda, L. y Adell, J. (2013). La anatomía de los PLE. En Castañeda, L. y Adell, J. (eds.), Entornos personales de aprendizaje: claves para el ecosistema educativo en red (pp. 11\#27). Marfil.

Cegarra, J. (2012). Fundamentos teórico epistemológicos de los imaginarios sociales. Cinta Moebio (43), 1\#13. http://www.mo ebio.uchile.cl/43/cegarra.htm

Corsi, G., Esposito, E. y Baraldi, C. (1996). Glosario sobre la teoría social de Niklas Luhmann. Anthropos, UIA, ITESO.

Espejo, R. (2012). Seeing organizations: epistemological considerations. Kybernetes. 41(3/3), 327\#338.

García, A. (2018). Educar para el asombro. Sencillez, confianza, paciencia y profundidad. Mensajero.

Gómez, D., García, F. y Therón, R. (2014). Analítica visual en e\#learning. El profesional de la información. 23(3), 236\#245. h ttps://doi.org/10.3145/epi.2014.may.03

Gros, B. (2008). Aprendizajes, conexiones y artefactos. La producción colaborativa del conocimiento. Editorial Gedisa.

Gros, B. y Mas, X. (2016). ¿Cómo aprender en red? En Gros, B. y Suárez\#Guerrero, C. (eds.), Pedagogía red. Una educación para tiempos de internet (pp. 55\#75). Octaedro; ICE Universidad de Barcelona.

Ison, R. (2017). Systems practice. How to act. In situations of uncertainty and complexity in a climate\#change world (2.. ed.). Springer Verlag.

Llorens, F. (2016) ¿Dónde aprender en internet? Territorialidad virtual y nuevas narrativas. En Gros, B. y Suárez\#Guerrero, C. (eds.), Pedagogía red. Una educación para tiempos de internet (pp. 135\#158). Octaedro; ICE Universidad de Barcelona.

Maas, M., Amozurrutia, J., Almaguer, P., González, L. y Meza, M. (2012). Sociocibernética, cibercultur@y sociedad. UNAM, Centro de Investigaciones Interdisciplinarias en Ciencias y Humanidades.

Maturana, H. y Varela, F. (1999). El árbol del conocimiento. Las bases biológicas del conocimiento humano. Debate.

Pintos, J. (1995). Los imaginarios sociales. La nueva construcción de la realidad social. Sal Terrae.

Pourdehnad, J., Warren, B., Wright, M. y Mairano, J. (2006). Unlearning/Learning Organizations - The Role of Mindset. Proceedings of the 50th Annual Meeting of the ISSS. Sonoma, CA, USA. http://journals.isss.org/index.php/proceedings50th/ar ticle/view/326/101 
Reyes, A. y Zarama, R. (1998). The process of emboying: a re\#construction of the process of learning. Cybernetics $y$ Human Knowing, .(3), 19\#33.

Sandoval, R. (2011). Las mediaciones tecnológicas en el campo educativo. Revista Educación y Humanismo, 13(21), 162 173. h ttps://doi.org/10.17081/eduhum.13.21.2269

Varela, F., Thompson, E. y Rosch, E. (1997). De cuerpo presente. Las ciencias cognitivas y la experiencia humana. Editorial Gedisa.

\section{Bibliografía consultada}

Pintos, J. (1999). Los imaginarios sociales del delito: La construcción social del delito a través de las películas (1930\#1999). http://idd00qmm.eresmas.net/articulos/delitocine .htm

Notas

1 Mediación tecnológica: uso de herramientas de tecnologías de información y comunicaciones que permiten interacciones entre los participantes de una comunidad, y a partir de experiencias cotidianas, son capaces de construir, retar y renovar sus saberes previos, para captar y aprovechar las oportunidades y así transformar la realidad (Adaptado de Sandoval, 2011).

2 Que revela e integra diferentes lecturas de una realidad alrededor de un objeto de estudio.

3 Transversalidad: que se extiende en diferentes aspectos. Transmedialidad: a través de diferentes medios disponibles.

4 https://www.mentimeter.com/

(c) (1) (3)(0) 